\title{
Diurnal variation in glycaemic response to a low- and high-glycaemic index (GI) mixed meal
}

\author{
M. Gibbs, D. Harrington and S. Hampton \\ Faculty of Health and Medical Sciences, University of Surrey, Guildford GU2 7XH, Surrey, UK
}

Diurnal variation in the postprandial hormone and metabolic responses has been well documented ${ }^{(1,2)}$. Research into the impact of this diurnal variation on the glycaemic response to high (HGI) and low GI (LGI) meals has been inconclusive. The present study investigates diurnal change in the GI of HGI and LGI macronutrient-matched meals. It was hypothesised that the glycaemic responses of HGI and LGI meals would be greater in the evening $(\mathrm{pm})$ compared with the morning $(\mathrm{am})$, and the glucose response prolonged after the pm meal. The HGI meal, when consumed as a pm meal, would give a significantly greater glycaemic response compared with the LGI meal.

In a randomised controlled single-blind cross-over study ten healthy subjects (nine female, one male; mean age 25.5 (SD 8.8 ) years and BMI 21.9 (SD 1.7) $\mathrm{kg} / \mathrm{m}^{2}$ ) were given a HGI or LGI meal at $0800 \mathrm{~h}$ or $2000 \mathrm{~h}$ on four separate occasions. Meal composition was matched for energy, carbohydrate, fat and protein. The GI of each meal was initially estimated by the method of Frost and Dornhurst ${ }^{(3)}$ from the published GI of the ingredients (HGI 72 and LGI 37). Subjects were fasted (minimum $8 \mathrm{~h}$ ) following a controlled LGI pre-meal before each leg of the study. Blood samples were taken using a finger-prick method at baseline (0 min) and 15, 30, 45, 60, 90 and 120 min postprandially. Samples were analysed for glucose using the YSI 2300 analyser (YSI (UK) Ltd, Fleet, Hants., UK). Comparison between meals and diurnal comparisons were made by repeated measures ANOVA with significance at $P=0.05$.

The $\mathrm{pm}$ area under the glycaemic response curves (mmol/l.min) was significantly higher than the corresponding am value for both meals: HGIam 218 (SE 33) v. HGIpm 351 (sE 44), $P=0.003$; LGIam 142 (SE 26) $v$. LGIpm 309 (SE 26), $P=0.0004$. There was a return to basal plasma glucose levels (mmol/l) at $2 \mathrm{~h}$ postprandially following both am meals (LGIam 4.99 (SE 0.29) and HGIam 4.83 (SE 0.24)), but there was no return to basal levels for either LGIpm or HGIpm by end of the study period (LGIpm 6.74 (SE 0.29) and HGIam 6.11 (SE $0.58)$ ). Comparison of the two am postprandial responses approached significance $(P=0.06)$; in contrast, pm postprandial responses were not significantly different $(P=0.29)$, suggesting any benefit of LGI in the morning has been lost as a result of the diurnal deterioration in glycaemic control.

The study demonstrates a clear diurnal variation in postprandial glycaemic response following consumption of LGI and HGI meals. This finding suggests that LGIpm meals are of less value in glycaemic control than LGIam and that consuming a main meal late in the day has an undesirable metabolic impact irrespective of the GI of the meal. The difference in the glycaemic responses $v$. calculated GI reflects a change in GI when consumed as a mixed meal, which support the findings previously reported ${ }^{(4)}$. The impact of this diurnal effect on subjects using LGI foods to aid glycaemic control, e.g. in subjects with type 2 diabetes, requires investigation.

1. Hampton SM, Morgan LM, Lawrence N et al. (1996) J Endocrinol 151, 259-267.

2. Ribeiro D, Hampton SM, Morgan LM et al. (1998) J Endocrinol 158, 305-310.

3. Frost G \& Dornhurst A (2000) Diabet Med 17, 336-345.

4. Gibbs M, Jetha A, Mellor D et al. (2006) Proc Nutr Soc 65, 82A. 